\title{
Towards Ergonomic Control of Collaborative Effort in Multi-human Mobile-robot Teams
}

\author{
Wansoo Kim ${ }^{1}$, Marta Lorenzini ${ }^{1,2}$, Pietro Balatti ${ }^{1,3}$, Yuqiang $\mathrm{Wu}^{1,4}$, and Arash Ajoudani ${ }^{1}$
}

\begin{abstract}
In this paper, we propose a control framework for a multi-human and mobile-robot collaborative team, that takes into account the co-workers' ergonomic requirements as well as the demand for high flexibility in the manufacturing industries. The new MObile Collaborative robotic Assistant (MOCA), which is composed of a lightweight manipulator arm, an underactuated hand, and a mobile platform driven by four omni-directional wheels enabling mobility in the workspace, is able to accomplish multiple tasks in a wide area with a high level of adaptability. In addition, an ergonomics module to anticipate and mitigate the human risk factors by means of a multi-object optimisation is integrated into the framework to ensure human safety and improvement of working conditions. The main advantage of this approach is that MOCA can assist multiple human operators, reducing their physical risks, with fast-adaptive capacities due to agile mobility and advanced interaction and manipulation. We validated the proposed method with an experiment simulating a simple manufacturing line which involves two subjects and the MOCA. The results demonstrate that the proposed framework is able to address multi-workers' ergonomics with a high level of flexibility in the workplace.
\end{abstract}

\section{INTRODUCTION}

In the last few years, a great part of the production flow in many industrial environments opened up to the concept of human-robot collaboration (HRC) whereby robots work alongside humans in close proximity. In HRC scenarios, where the robot and the human are coupled to accomplish a task, the robot power generation capacity can be integrated with human's cognitive skills and experience [1]. As a result, robots can reduce effort and fatigue, and prevent injuries by providing assistance to humans [2]. Accordingly, co-carrying [3], [4], and co-manipulation [5] have been proposed among the applications of HRC, to name a few.

The key factor to ensure the success of collaborative human-robot synergies is safety, primarily from the human point of view [6]. The existing HRC frameworks are potentially capable of demonstrating an effective physical support to the human, however, the human safety here is guaranteed by restricting the interaction strategies and avoiding an actual coupling between the human and the robot. Indeed, common strategies to ensure the human safety in HRC are built around the concepts of power-limitation (according to the existing

${ }^{1} \mathrm{HRI}^{2}$ Laboratory, Istituto Italiano di Tecnologia, Genoa, Italy, Email: wan-soo.kim@iit.it

${ }^{2}$ Department of Electronics, Information and Bioengineering, Politecnico di Milano, Milano, Italy.

${ }^{3}$ Dept. of Information Engineering, University of Pisa, Italy.

${ }^{4}$ State Key Laboratory for Manufacturing System Engineering, Shaanxi Key Laboratory of Intelligent Robots and School of Mechanical Engineering, Xi'an Jiaotong University, China

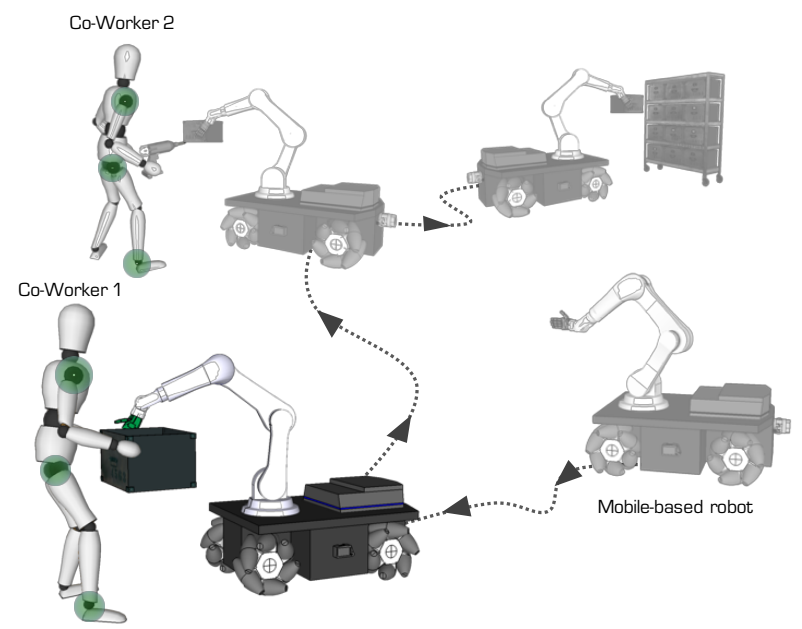

Fig. 1: The proposed multi-human mobile-robot control framework aims to improve workers' ergonomics by integrating a multi-objective optimisation into a mobile collaborative robot assistant.

standards, e.g., ISO/TS 15066 [7]) or accidental collision avoidance [8], [9]. As a result, collaborative robots of today mostly 'co-exist' with human partners, and do not provide a personalised level of assistance.

One of the first attempts to bring HRC to the level of subject-specific, assistive collaboration was made by our recent works, which enabled robot adaptation to the variability of the task as well as human dynamic states [4], [10]. This concept was then exploited in similar settings [11]-[13], with the aim to contribute to the reduction of work-related musculoskeletal disorders (WMSD), the single largest category of work-related injuries and responsible for almost the 30\% of all worker's compensation costs [14]. In recent times, researchers in this field are aiming at approaches for the realtime assessment of human ergonomics in the workplace. In fact, in [11] contextual ergonomics models were proposed, which relied on training with full musculoskeletal simulations but could work in a low-dimensional space and thus demonstrated real-time capabilities. An activity recognition algorithm was presented in [12] to infer the actions and postures that are considered in well-established ergonomic worksheets and thus to develop an automatic ergonomics assessment system. Finally, the human body posture was optimised in [13] using postural assessment techniques, and a personalized human kinematic model, by following the guidance of a robot and a visual feedback interface.

To address workers' ergonomics evaluation even from a dynamic point of view, we recently proposed a method to estimate in real-time the loadings on the human body joints 


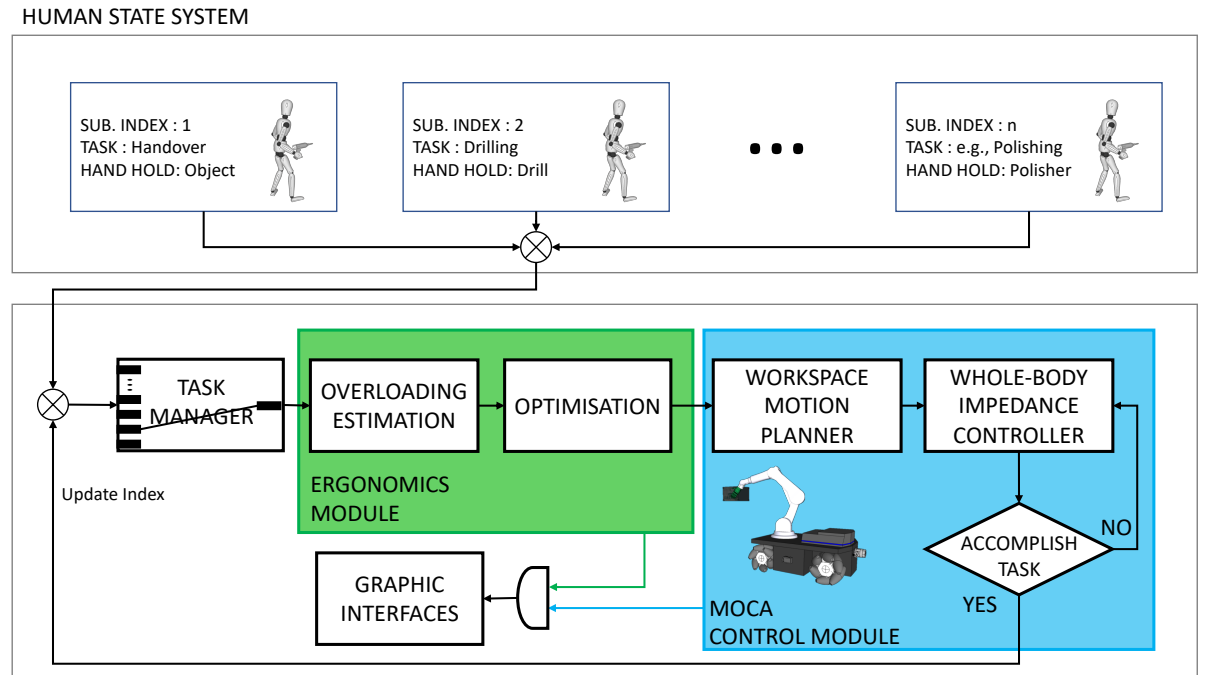

ROBOT STATE SYSTEM

(a)

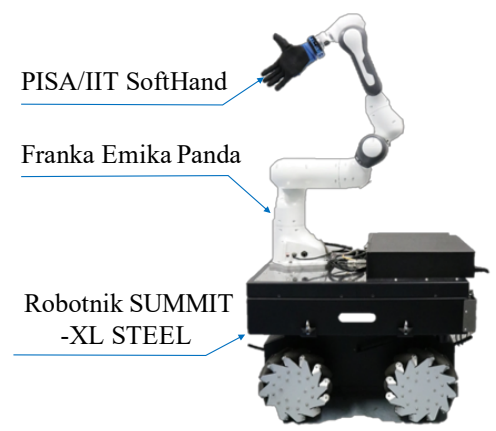

(b)

Fig. 2: (a) The overall control architecture of the presented framework and (b) the MObile Collaborative robotic Assistant (MOCA).

due to the external forces [15], based on the variations of the human centre of pressure $(\mathrm{CoP})$ and the ground reaction force (GRF). Then, we developed a HRC framework to mitigate such a risk factor, in which a robot guided the human partner toward an optimised posture to perform a heavy manipulation task in a more ergonomic way [4]. Such a framework was further improved in [10], integrating reconfigurability and adaptation capacities to the human intentions and the workspace. Nevertheless, the proposed approach lacked flexibility because of the fixed base of the robot, hence, in real industrial scenarios including multiple workers which operate in a wide area, its applicability may be limited.

To increase the potential of the collaborative robotic technologies in the workplace, while addressing the demands of frequently changing production processes, the concept of mobility must be integrated into the robot control framework (see Fig.1). Accordingly, the focus of this work is first, to develop a new MObile Collaborative robotic Assistant (MOCA) to combine agile mobility with advanced interaction and manipulation. Next, we develop a multi-objective optimisation function to ensure robot adaptation to multiple human workers' kinodynamic states and variable tasks. This is achieved by loco-manipulation control of MOCA, where the robot whole-body optimised movements contribute to a better human manipulation and to the reduction of human joint torque overloadings.

To validate the proposed strategy, we simulate a manufacturing line where two human subjects perform handover and drilling tasks, respectively. MOCA moves in the workspace and assists each worker individually, adapting its behaviour according to the subject-specific ergonomic factors and the task, to guide them to a more ergonomic body configuration.

\section{Ergonomic Human-Robot Team}

Fig. 2 represents the overall schema of the proposed framework. The control architecture is characterised by a modular structure (see Fig. 2a) including: a human-ergonomics module, a mobile-based manipulation control module, and an integration module. The versatile mobile-based assistive robot, MOCA, is developed to address the mobility requirement in manufacturing lines (see Fig. 2b). Each module is described in details in the following sections.

\section{A. MOCA Control Module}

This module is responsible for the control of MOCA platform. The "workspace motion planner" unit receives as input the pose in Cartesian space to be reached by MOCA end-effector, previously computed by the "optimisation" unit (see Fig. 2a). Fifth-order polynomial trajectories are designed to reach the desired pose, given as input to the "whole-body impedance controller" unit, in order to achieve smooth robot movements.

MOCA [16] is a new versatile research robotic platform, designed for advanced research on human-robot interaction and collaboration, with potential applications in flexible manufacturing scenarios. Three commercial components are assembled to build it: a Panda robotic arm by Franka Emika, equipped with the underactuated Pisa/IIT SoftHand [17], that are mounted on top of a SUMMIT-XL STEEL mobile platform by Robotnik. The control interfaces of these components are respectively torque-based, current-based (underactuated), and velocity-based. Considering the mobility and the safety during navigation and physical interaction, a whole-body impedance controller is developed based on the assumptions of whole-body dynamics.

As it is well known, the impedance controller architecture is employed to generate torques commands. However, the control interface of the mobile platform is velocity-based. Therefore, an admittance controller is implemented as following:

$$
\mathbf{M}_{a d m} \ddot{\mathbf{q}}_{v}^{d e s}+\mathbf{D}_{a d m} \dot{\mathbf{q}}_{v}^{d e s}=\boldsymbol{\tau}_{v}^{v i r}+\boldsymbol{\tau}_{v}^{e x t},
$$


where $\mathbf{M}_{a d m} \in \mathbb{R}^{3 \times 3}$ and $\mathbf{D}_{a d m} \in \mathbb{R}^{3 \times 3}$ are the virtual inertial and virtual damping terms, $\dot{\mathbf{q}}_{v}^{\text {des }} \in \mathbb{R}^{3}$ is the desired velocity of translation in $x$ and $y$ direction and rotation around $z$ direction sent to the mobile platform, $\tau_{v}^{v i r} \in \mathbb{R}^{3}$ and $\tau_{v}^{\text {ext }} \in \mathbb{R}^{3}$ are the virtual and external torques.

Although mobile manipulators like MOCA offer a high potential for flexible automation, at the same time they raise a number of challenges, as introduced in [18]. The greatest challenge is the existence of dynamic interactions between the manipulator and the mobile platform, which may cause instability problems for the coupled system [19]. Considering the experiment introduced in Sec. III we plan to perform, the following reasonable assumptions are introduced:

Assumption 1: The gain of the low level velocity controller for the mobile platform is high enough to realise arbitrary desired trajectories and compensate any dynamic disturbances from the external.

Assumption 2: During the experiment, the movement of the mobile platform is smooth enough. Hence, its motion does not have much effect on the upper manipulator.

Based on Assumption 1, i.e. $\ddot{\mathbf{q}}_{v} \approx \ddot{\mathbf{q}}_{v}^{\text {des }}$. Combined with Assumption 2, a decoupled whole-body dynamic formula is obtained as following:

$$
\begin{aligned}
& \left(\begin{array}{cc}
\mathbf{M}_{a d m} & \mathbf{0} \\
\mathbf{0} & \mathbf{M}_{r}
\end{array}\right)\left(\begin{array}{c}
\ddot{\mathbf{q}}_{v} \\
\ddot{\mathbf{q}}_{r}
\end{array}\right)+\left(\begin{array}{cc}
\mathbf{D}_{a d m} & \mathbf{0} \\
\mathbf{0} & \mathbf{C}_{r}
\end{array}\right)\left(\begin{array}{c}
\dot{\mathbf{q}}_{v} \\
\dot{\mathbf{q}}_{r}
\end{array}\right)+\left(\begin{array}{c}
\mathbf{0} \\
\mathbf{g}_{r}
\end{array}\right) \\
& =\left(\begin{array}{c}
\boldsymbol{\tau}_{v}^{\text {vir }} \\
\boldsymbol{\tau}_{r}
\end{array}\right)+\left(\begin{array}{c}
\boldsymbol{\tau}_{v}^{\text {ext }} \\
\boldsymbol{\tau}_{r}^{\text {ext }}
\end{array}\right) .
\end{aligned}
$$

The first line represents the dynamics of the mobile platform as shown in (1) while the second line describes the dynamics of the manipulator, where $\mathbf{q}_{r} \in \mathbb{R}^{7}$ is the joint coordinates array, $\mathbf{M}_{r} \in \mathbb{R}^{7 \times 7}$ is the symmetric and positive definite inertial matrix, $\mathbf{C}_{r} \in \mathbb{R}^{7 \times 7}$ is the Coriolis and centrifugal matrix, $\mathbf{g}_{r} \in \mathbb{R}^{7}$ is the gravity vector, $\boldsymbol{\tau}_{r} \in \mathbb{R}^{7}$ and $\boldsymbol{\tau}_{r}^{\text {ext }} \in \mathbb{R}^{7}$ are the commanded torques and external torques of the arm, respectively.

Based on the simplified whole-body dynamics, the wholebody impedance controller is constructed according to the method elaborated in [20]. The stability properties of this controller can be easily analysed since a decoupled wholebody dynamics is obtained [19]. The Cartesian impedance controller input for the main task with Cartesian error $\tilde{\mathbf{x}}=$ $\mathbf{x}-\mathbf{x}_{d}$ is as following 1

$$
\boldsymbol{\tau}_{i m p}=\mathbf{J}_{w}^{T}(\mathbf{q})\left(\boldsymbol{\Lambda}_{w}(\mathbf{x}) \ddot{\mathbf{x}}_{d}+\mu_{w}(\mathbf{x}, \dot{\mathbf{x}}) \dot{\mathbf{x}}_{\mathbf{d}}-\mathbf{K}_{d} \tilde{\mathbf{x}}-\mathbf{D}_{d} \dot{\tilde{\mathbf{x}}}\right),
$$

where $\mathbf{x} \in \mathbb{R}^{6}$ and $\mathbf{x}_{d} \in \mathbb{R}^{6}$ are the actual and desired wholebody Cartesian position and orientation w.r.t. the global reference frame $\Sigma_{W}, \mathbf{K}_{d} \in \mathbb{R}^{6 \times 6}$ and $\mathbf{D}_{d} \in \mathbb{R}^{6 \times 6}$ are the desired Cartesian stiffness and damping matrices, $\mathbf{J}_{w}(\mathbf{q}) \in \mathbb{R}^{6 \times 10}$ is the whole-body Jacobian matrix, $\boldsymbol{\Lambda}_{w}(\mathbf{x})$ and $\mu_{w}(\mathbf{x}, \dot{\mathbf{x}})$ denote the whole-body Cartesian inertial, Coriolis and centrifugal matrices, respectively.

\footnotetext{
${ }^{1}$ Gravity force of the robotic arm is compensated by the inner controller of Franka Emika Panda.
}

\section{B. Ergonomics Module}

We recently proposed a human-robot collaboration framework to improve human ergonomics by using a collaborative robot [4]. The method was based on a real-time estimation function for the human co-worker's body joint overloading effects, which are caused by the external forces (e.g. tools or objects). The excessive human joint overloadings were then mitigated through the assistance of the collaborative robot, which guided the human toward an optimised body configuration, which was aiming to reduce the risk of joint injuries.

Accordingly, the ergonomics module included in the proposed HRC framework will exploit the same strategy to address human ergonomics for multiple tasks. Such an ergonomics module is composed by an "overloading estimation" and an "optimisation" unit (see Fig. 2a). This section provides a brief overview of the algorithm. A more exhaustive explanation can be found in [4].

The overloading joint torques are estimated from the difference between the human joint torques computed with and without the effect of the external forces. In this method, a reduce-complexity human model is exploited to ensure the real-time applicability of the approach, taking into account the body joints which are mainly at risk of injuries. Based on a floating base human model, the generalised coordinates of the system are defined as $\mathbf{q}_{h}=\left[\begin{array}{lll}\mathbf{x}_{0}^{T} & \boldsymbol{\theta}_{0}^{T} & \mathbf{q}_{h_{a}}^{T}\end{array}\right]^{T} \in \mathbb{R}^{6+n}$, where $n$ is the number of DoF in the model, $\mathbf{x}_{0} \in \mathbb{R}^{3}$ and $\boldsymbol{\theta}_{0} \in \mathbb{R}^{3}$ represents the position and orientation of $\Sigma_{0}$ w.r.t world reference frame $\Sigma_{W}$, while $\mathbf{q}_{h_{a}}$ are the angular positions of human joints.

When the effect of external forces is not considered, the force applied at the contact point $\operatorname{CoP} \mathbf{C}_{P_{w o}}$ is represented by just the vertical GRF $\mathbf{f}_{w o}$ (obtained from the human body mass). $n_{f}$ is the number of contact points with the ground and the condition $n_{f} \leq 2$ is valid. Hence, the human joint torques without considering the external forces can be represented as

$$
\mathbf{M}\left(\mathbf{q}_{h}\right) \ddot{\mathbf{q}}_{h}+\mathbf{C}\left(\mathbf{q}_{h}, \dot{\mathbf{q}}_{h}\right) \dot{\mathbf{q}}_{h}+\mathbf{G}\left(\mathbf{q}_{h}\right)=\mathbf{S}^{T} \boldsymbol{\tau}_{w o}+\sum_{i=1}^{n_{k}} \mathbf{J}_{C_{P_{w o}}}^{T}\left(\mathbf{q}_{h}\right) \mathbf{f}_{w o, i},
$$

where $\mathbf{M}, \mathbf{C}$, and $\mathbf{G}$ represent the inertia matrix, the centrifugal and Coriolis forces, and the vector of the gravity force, respectively. In addition, $\mathbf{S}=\left[\begin{array}{ll}\mathbf{0}_{n \times 6} & \mathbf{I}_{n \times n}\end{array}\right] \in \mathbb{R}^{n \times(n+6)}$ is the actuation matrix, $\boldsymbol{\tau}_{w o} \in \mathbb{R}^{n}$ represent the joint torques without the external forces, and $\mathbf{J}_{C_{P_{w o} i}}\left(\mathbf{q}_{h}\right)$ is the contact Jacobian at $i$-th CoP. On the other hand, when the interaction force $\mathbf{f}_{h}$ is applied at the point $\mathbf{a}_{h}$ in the same configuration $\mathbf{q}_{h}$, the new position of the CoP is $\mathbf{C}_{P_{w t}}$ and $\mathbf{f}_{w t}=\mathbf{f}_{w o}+\mathbf{f}_{h}$ is the force applied at the ground. The human joint torques $\tau_{w t} \in \mathbb{R}^{n}$ considering the interaction forces can then be expressed as

$$
\begin{aligned}
& \mathbf{M}\left(\mathbf{q}_{h}\right) \ddot{\mathbf{q}}_{h}+\mathbf{C}\left(\mathbf{q}_{h}, \dot{\mathbf{q}}_{h}\right) \dot{\mathbf{q}}_{h}+\mathbf{G}\left(\mathbf{q}_{h}\right)= \\
& \mathbf{S}^{T} \boldsymbol{\tau}_{w t}+\sum_{i=1}^{n_{f}} \mathbf{J}_{C_{P_{w t}}}^{T}\left(\mathbf{q}_{h}\right) \mathbf{f}_{w t, i}-\sum_{j=1}^{n_{h}} \mathbf{J}_{a_{h j}}^{T}\left(\mathbf{q}_{h}\right) \mathbf{f}_{h, j}
\end{aligned}
$$

$\mathbf{J}_{a_{h j}}\left(\mathbf{q}_{h}\right)$ is the contact Jacobian at $\mathbf{a}_{h}$ and $n_{h}$ are the number of contact points at the hand, with the condition $n_{h} \leq 2$. 
The difference between (4) and (5) represents the overloading joint torque concept. Since the matrices $\mathbf{M}, \mathbf{C}$, and $\mathbf{G}$ are the same in the two conditions, they can be cancelled, lightening the computations. The overloading joint torques considering the force distribution ratios $\left(0 \leq \eta_{j}, \zeta_{i} \leq 1\right.$ [21]) can then be defined as

$\Delta \boldsymbol{\tau}_{s}=-\sum_{j=1}^{n_{h}} \mathbf{J}_{a_{h j}}^{T}\left(\mathbf{q}_{h}\right) \eta_{j} \mathbf{f}_{h}+\sum_{i=1}^{n_{f}}\left(\mathbf{J}_{\Delta C_{P_{w i}}}^{T}\left(\mathbf{q}_{h}\right) \mathbf{f}_{w t, i}+\mathbf{J}_{C_{P i}}^{T}\left(\mathbf{q}_{h}\right) \zeta_{i} \mathbf{f}_{h}\right)$,

where the Jacobian of the CoP displacement is defined as $\mathbf{J}_{\Delta C_{P_{w} i}}=\mathbf{J}_{C_{P_{w o} i}}-\mathbf{J}_{C_{P_{w t} i}}$. By means of the overloading joint torques, the dynamic states can be provided for any arbitrary human motion when interaction forces are applied.

To obtain the whole-body $\mathrm{CoP} \mathbf{C}_{P_{w o}}$ and $\mathbf{C}_{P_{w t}}$, we use a statically equivalent serial chain (SESC) technique ${ }^{2}$ and we take advantage of the knowledge about the predefined tools parameters, based on [23]. In addition to enabling the estimation of $\mathbf{C}_{P_{w o}}$ in real-time, the method described in [23] allows to obtain the $\mathbf{C}_{P_{w t}}$ when the properties of an external objects mass $m_{e}$ and centre of mass position are known. The ergonomics module contain data about the predefined tool parameters due to the production process.

To guide the human toward a more ergonomic condition during HRC, the collaborative robot assumes an optimised configuration computed by means of an optimisation procedure proposed in a previous study [4]. The aim of the optimisation is to find the human posture which minimises the overloading joint torques following certain constraints. To manage a multi-tasks scenario, the optimisation module has to modify the optimisation constraints on the basis of the task requirements. Such task-dependent constraints are selected by the "task manager" unit, whose role will be explained in the next section.

The optimisation general objective can be defined as

$$
\min _{\mathbf{q}_{h}}\left\|\Delta \tau^{T} \mathbb{W} \Delta \tau\right\|
$$

where $\mathbb{W}=\operatorname{diag}\left[\begin{array}{lll}\Delta \tau_{1} / \tau_{\max _{1}} & \cdots & \Delta \tau_{n} / \tau_{\max _{n}}\end{array}\right] \in \mathbb{R}^{n \times n}$, with $\tau_{\text {max }_{n}}$ being obtained by human biomechanical data [24], and $\mathbb{W}$ is a weight factor matrix to assign higher priorities to the highly overloaded body joints. The general constraints to ensure the safety and the stability aspects based on [4] are assigned by means of the joint angle boundaries and the support polygon, respectively. Instead, the task-specific constraint regarding arm manipulability can be implemented as described in [23].

The human end-point manipulability represents an index concerning the manipulation capacity to produce the velocity and force at the limb end-point in different configurations. The manipulability aspect can be defined as $w=$ $\left|\operatorname{det}\left(\mathbf{J}_{a}\left(\mathbf{q}_{a}\right)\right)\right|$, where $\mathbf{J}_{a}$ is the arm Jacobian, addressing the ratio between the eigenvalues of the manipulability ellipsoid. For example, if the task required a complex manipulation including multi-directional motions (e.g. drilling, polishing, etc.), the manipulability ellipsoid would be designed to

\footnotetext{
${ }^{2}$ Details about this method can be found in [22].
}

have an isotropic shape (i.e. $w \approx 1$ ) in the task space. In summary, the optimisation module selects the constraints which correspond to the considered task, then, it provides the optimised human body configuration, preventing any excessive joint loading and maximising the efficiency of the task performance.

\section{Integrated Framework}

Fig. 2a illustrates the overall control architecture of the proposed framework to perform ergonomic control of a multi-human mobile-robot collaborative team. The control flow consists of two main parts: the human state system and the robot state system. The human state system aims first at measuring $n$ multi-humans' motion by means of motion tracking devices in real-time. Next, it sends the motion measurements and the user-specific data to the robot state system. The user-specific data includes the subject parameters (here, the CoP model parameters) and the predefined task information (i.e. the target of the task and the relevant tool parameters, etc.).

On the other hand, the robot state system aims at integrating the modules explained in the previous sections and at allocating the task execution to MOCA via the task manager. The status of task execution is represented by the graphic interface, providing a visual feedback to the human coworkers. The control flow is initiated with the setting of the human state system, then the task manager is initialised with the first subject index, and connected to the ergonomics module which provides the motion measurements and the userspecific data of the first subject. As soon as the optimised configuration of the considered subject is obtained by the ergonomics module, the worker's task execution is facilitated in Cartesian space by the guidance of MOCA end-effector movements. In fact, MOCA control module is employed to convert the optimised human pose into the desired position of the end-effector. The "workspace motion planner" unit then generates the path that the robot joints need to follow to approach the desired point, by means of the "wholebody impedance controller". When the task is completed, the subject index is updated in the "task manager" unit and the second task is initiated. A proper communication is set between the state systems and the modules are connected between each other via ROS communication protocol, based on ROS topics.

\section{EXPERIMENTAL VALIDATION OF THE FRAMEWORK}

\section{A. Experimental Setup}

In this section, we first describe the procedure to evaluate the proposed method through a sEMG analysis. Next, we present an experiment which demonstrates the functioning of the whole framework. The whole experimental procedure was approved by the ethics committee Azienda Sanitaria Locale Genovese (ASL) N.3 (Protocol IIT_HRII_001).

Two human subjects (1 [female] and 2 [male]) were involved in the experimental session. Both of them wore a MVN Biomech suit (Xsens Tech) provided with seventeen inter-connected inertial measurement unit (IMU) sensors 


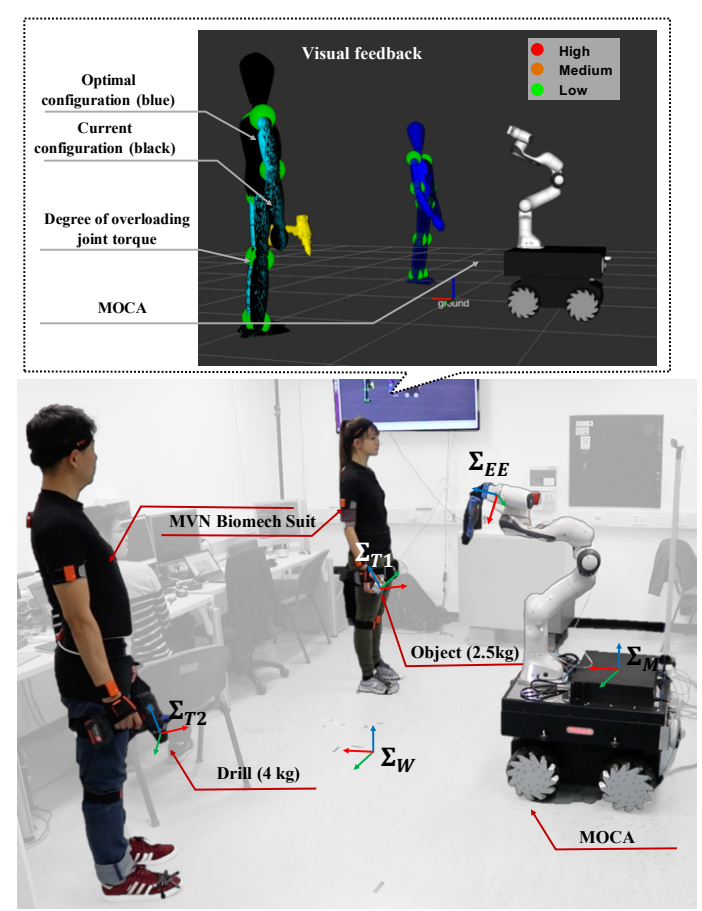

Fig. 3: Overview of the experimental setup: two subjects and MOCA were involved in the experiment (bottom). The visual feedback provided to the subjects during the experiment (top).

to measure the whole-body motion. Two different tasks were defined for the subjects: subject 1 had to perform a handover task, passing an object to MOCA, while subject 2 had to perform a drilling task on the object held by MOCA. For each subject the algorithm computed the optimal body configuration to perform the specific task, by means of the procedure described in Sec.II. Since different tasks were defined, different constraints were considered for the optimisation.

For subject 1, the constraints concerned the human support polygon and the shared work space between the human subject and MOCA. For subject 2, the constraints of the optimisation concerned the human support polygon and the human manipulability. To prove the benefit of the proposed method, we measured the human muscle activity in the optimal body configuration and compared the results to other non-optimal ones. The objective was to show that, in the computed optimal posture, the human effort was lower than in other postures, thus the risk of injuries was reduced. Accordingly, we measured the muscle activity through the sEMG signals of some significant muscles, in the optimal body configuration and in other two configurations. Such configurations were defined considering the human hand located $20 \mathrm{~cm}$ ahead $\left(P_{A}\right)$ and behind $\left(P_{B}\right)$, respectively, of the human hand in the optimal configuration $\left(P_{O p t}\right)$. Six sEMG sensors were placed on the arm of each subject, specifically on the following muscles: the anterior deltoid $(\mathrm{AD})$, the posterior deltoid (PD), the biceps (BC), the triceps (TC), the flexor carpi radialis (FC), the extensor carpi radialis (EC). Next, each configuration was maintaned for 10 seconds by the subjects, with the necessary rest time in between. Meanwhile, the sEMG data was collected. The measured
TABLE I: Experimental results of the optimisation function compared between two tasks. The results are divided in three different configurations for the overloading joint torques, muscle activity, and the manipulability aspect.

\begin{tabular}{|c|c|c|c|c|c|c|c|}
\hline \multirow[b]{2}{*}{$P_{H}$} & & \multicolumn{3}{|c|}{ Subject 1} & \multicolumn{3}{|c|}{ Subject 2} \\
\hline & & ${ }^{1} P_{O p t}$ & ${ }^{1} P_{A}$ & ${ }^{1} P_{B}$ & ${ }^{2} P_{O p t}$ & ${ }^{2} P_{A}$ & ${ }^{2} P_{B}$ \\
\hline$\overline{\overline{\Delta \Delta \tau_{H}}}$ & & $\overline{5.71}$ & 7.92 & 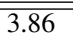 & 12.49 & 21.88 & 4.89 \\
\hline$\Delta \tau_{H}$ & & 5.86 & 8.19 & 3.72 & 13.66 & 22.75 & 3.95 \\
\hline$\Delta \tau_{H}$ & {$[\mathrm{Nm}]$} & 6.32 & 8.68 & 3.22 & 14.96 & 24.15 & 3.42 \\
\hline$\Delta \tau_{H}$ & & 4.32 & 6.51 & 1.63 & 8.88 & 17.55 & 0.92 \\
\hline$\Delta \tau_{H}$ & & 5.09 & 5.26 & 0.50 & 11.55 & 12.77 & 3.93 \\
\hline$A D$ & & 3.71 & 68.89 & 9.93 & 6.50 & 59.14 & 15.07 \\
\hline$P D$ & & 6.79 & 2.64 & 32.96 & 8.18 & 2.19 & 24.35 \\
\hline$B C$ & [\%] & 5.78 & 29.13 & 19.05 & 41.12 & 58.74 & 25.11 \\
\hline$T C$ & & 21.71 & 34.75 & 30.03 & 16.53 & 16.16 & 35.52 \\
\hline$F C$ & & 5.51 & 26.66 & 26.62 & 7.54 & 9.88 & 7.81 \\
\hline$E C$ & & 21.55 & 10.86 & 21.13 & 27.43 & 9.91 & 39.79 \\
\hline$w$ & [\%] & - & - & - & 95.17 & 68.89 & 64.34 \\
\hline
\end{tabular}

sEMG data was filtered and normalised.

The experimental setup of the second experiment is illustrated in Fig. 3. The actors involved were MOCA and the two human subjects. Each human subject was wearing the MVN Biomech suit. A global reference frame $\Sigma_{W}$ was defined on the floor of the experiment room to localise the reference frame of each actor involved. Both MOCA and the MVN Biomech suits worn by the human subjects were calibrated so as to set $\Sigma_{W}$ as their global reference frame. $\Sigma_{T 1}, \Sigma_{T 2}$ and $\Sigma_{M}$ were the local reference frames of subject 1 , subject 2 and MOCA, respectively. The experimental procedure included two phases: the handover task performed by subject 1 and the drilling task performed by subject 2 . Both the human subjects in the two phases had to accomplish their own task in the body configuration which resulted from the optimisation procedure described in Sec.II. During the first phase, subject 1 held an object and MOCA approached her assuming a configuration that guided her to the optimal body posture to hand over the object to MOCA. During the second phase, MOCA approached subject 2 offering him the object which he had to drill, in such a way that he could assume the optimal body configuration to perform the task.

\section{B. Results}

The results of the first experiment for the evaluation of the proposed method are shown in Table I. We represent here the overloading joint torques and muscle activity of subject 1 and 2 , in the optimised $\left(P_{O p t}\right)$ and unoptimised positions $\left(P_{A}\right.$ and $\left.P_{B}\right)$, respectively. The manipulability aspect, which is considered among the constraints in the optimisation for the drilling task, is also reported for subject 2.

Considering the handover task (subject 1), the sum of overloading torques in all the body joints, computed in the examined configurations, were: $27.30 \mathrm{Nm}$ in ${ }^{1} P_{O p t}, 36.55$ $\mathrm{Nm}$ in ${ }^{1} P_{A}$, and $12.92 \mathrm{Nm}$ in ${ }^{1} P_{B}$. On the other hand, the muscular effort in the arm (i.e. mean value between all the muscles of the normalised muscle activity) was: 10.84 $\%$ in ${ }^{1} P_{O p t}, 23.82 \%$ in ${ }^{1} P_{A}$, and $23.28 \%$ in ${ }^{1} P_{B}$. In the optimised configuration ${ }^{1} P_{O p t}$, the overloading joint torques were higher than in ${ }^{1} P_{B}$ but muscle activity was fairly lower than in the other positions. It worth to mention that the optimisation constraints associated with human muscle prop- 

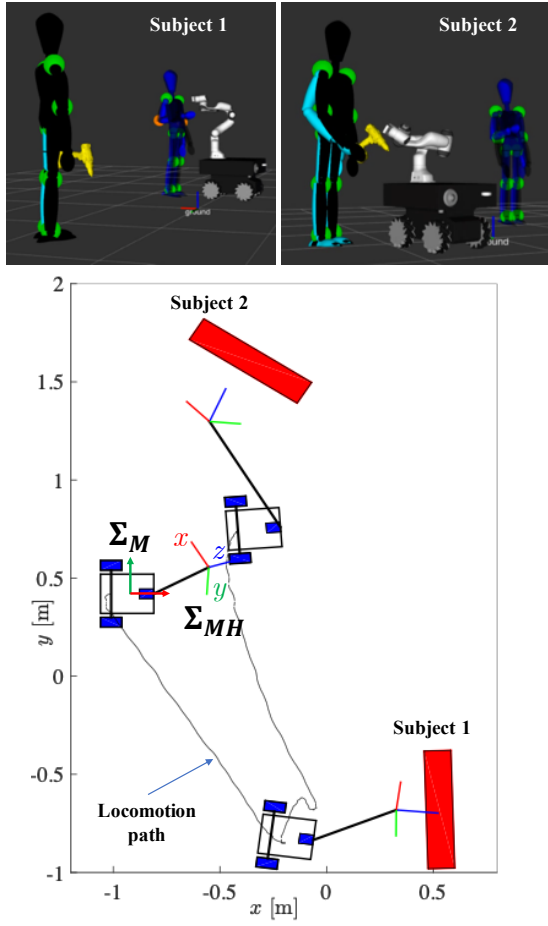

(a)
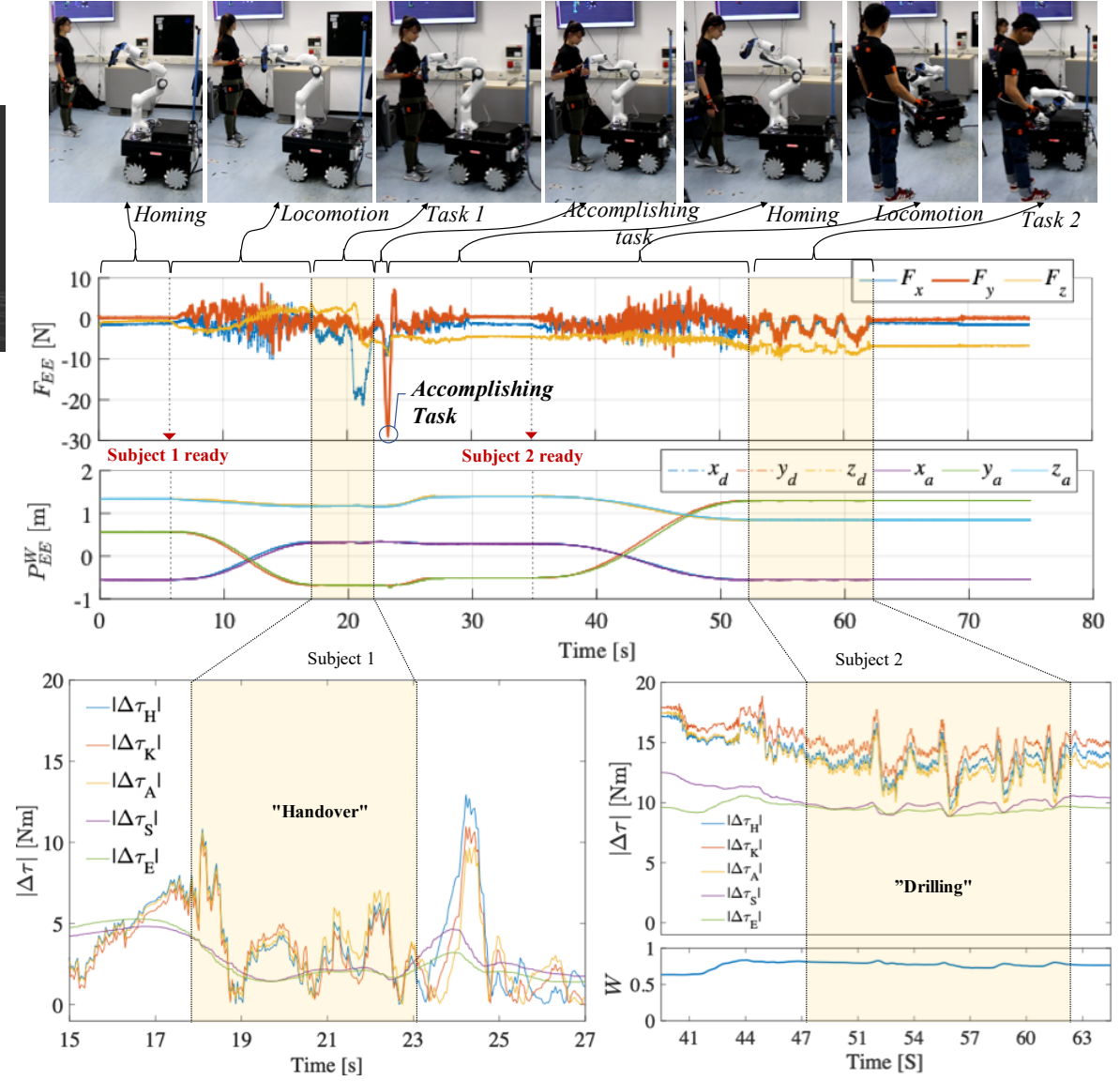

(b)

Fig. 4: Experimental results of the multi-human mobile robot team executing simple manufacturing tasks. (a) The trajectory of the mobile platform during the execution of two tasks by the subjects (bottom) and the visual feedback provided to the subjects (top). (b) The interaction forces and the position of MOCA end-effector are illustrated in the first two rows, respectively, and the overloading joint torques and manipulability (only subject 2 ) estimated by the ergonomics module for each subject are illustrated in the bottom.

erties (i.e. the range of motion of the joints), reached their boundaries in proximity to ${ }^{1} P_{A}$, hence, our "optimisation" unit tried to avoid such a configuration which represented a singularity. Considering the drilling task (subject 2), the sum of overloading torques in all the body joints, computed in the examined configurations, were: $61.52 \mathrm{Nm}$ in ${ }^{2} P_{O p t}$, $99.11 \mathrm{Nm}$ in ${ }^{2} P_{A}$, and $17.11 \mathrm{Nm}$ in ${ }^{2} P_{B}$. The muscular effort in the arm were instead: $21.46 \%, 31.20 \%$, and 29.53 $\%$ in ${ }^{2} P_{O p t},{ }^{2} P_{A}$, and ${ }^{2} P_{B}$, respectively. Furthermore, the manipulability aspect was computed and is here reported in percentage. A high value of manipulability means that the manipulability ellipsoid is nearly isotropic. The values computed were: $95.17 \%, 68.89 \%$, and $64.34 \%$ in ${ }^{2} P_{O p t}$, ${ }^{2} P_{A}$, and ${ }^{2} P_{B}$, respectively. The muscle activity and the overloading joint torques presented similar results to the ones of the previous subject, however, in ${ }^{2} P_{O p t}$ a higher capacity in terms of tool manipulation can be achieved by subject 2. This can be explained by the fact that the optimisation procedure attempted to minimise the overloading effect while maximinsing manipulability.

The results of the multi-human MOCA team experiment are illustrated in Fig.4. The optimised postures of each subject and the configuration changes of MOCA, provided by the visual feedback module, are illustrated in the first row of Fig. 4a. The bottom graph depicts the positions of the two subjects in the work space while performing their task and illustrates the relevant trajectories (position and orientation) that MOCA followed to guide the two subjects toward the optimised task executions. Fig. $4 \mathrm{~b}$ represents the whole sequence of tasks execution considering both the robot and the subjects points of view. The first two plots show the external interaction forces and positions, which were measured at MOCA end-effector w.r.t $\Sigma_{W}$. The variations of the overloading joint torques are represented in the bottom plot of Fig.4b, for each subject during the execution of their task. For subject 2, the value of the manipulability aspect throughout the experiment is also depicted. In the homing phase, the robot waited for the first task to start. Then, as soon as optimisation was performed, the locomotion phase was triggered and the robot was induced to move towards the first optimised configuration for picking up the object from subject 1 . When the task finished, the robot was notified about the task accomplishment: the robot end-effector was pushed by the subject with a force overcoming a pre-defined threshold $\left(F_{y} \geq 20 N\right)$. It should be noted, in this respect, that the increment of the overloading joint torques at the end of the task was due to the human act of pushing the robot. Subsequently, the robotic arm (but not mobile platform) came 
back to the home configuration and MOCA waited for the next task. Similarly, when optimisation was performed, the locomotion phase started and the robot was induced to move towards the optimised configuration for offering the object to subject 2, which had then to operate on it. During the drilling task, the manipulability index maintained a high value, around $83.45 \%$, which means that the manipulability ellipsoid became nearly isotropic (bottom in right plot of Fig.4b).

\section{CONClusions And Future Work}

In this paper we developed a novel HRC control framework to account for the assessment of multi-human ergonomics in the workplace. The proposed framework was able to mitigate the risk of injuries for multiple humans performing different tasks in a wide working area, contributing to a substantial improvement in the flexibility of the HRC solutions in industry. This was achieved by integrating robot loco-manipulation control into a multi-objective optimisation framework, to improve robot adaptation to the variability of the task as well as human workers. A simple manufacturing line was simulated in this study, where two human subjects performed a handover and drilling task, respectively, collaborating with a recently developed mobile collaborative robot, MOCA. A task-dependent optimisation was accomplished by the ergonomics module, and its effectiveness was validated by means of the sEMG measurements, torque variations, and consideration on the manipulability aspect. The optimised ergonomic configurations were conveyed to the subjects according to the their task by the whole-body impedance controller of MOCA.

The key advantage of the proposed strategy lies in its applicability to the realistic industrial environments which demand for high flexibility in the few-of-a-kind production processes. Future work will focus on further improvement of the reconfiguration capabilities of the framework, by developing a system to automatise the task management, relying on a vision system which performs the real-time tracking of the human and tools recognition in multi-subject, multi-task scenarios.

\section{REFERENCES}

[1] A. Ajoudani, A. M. Zanchettin, S. Ivaldi, A. Albu-Schäffer, K. Kosuge, and O. Khatib, "Progress and prospects of the human-robot collaboration," Autonomous Robots, Oct 2017. [Online]. Available: https://doi.org/10.1007/s10514-017-9677-2

[2] O. Khatib, K. Yokoi, O. Brock, K. Chang, and A. Casal, "Robots in human environments: Basic autonomous capabilities," The International Journal of Robotics Research, vol. 18, no. 7, pp. 684-696, 1999.

[3] D. J. Agravante, A. Cherubini, A. Bussy, P. Gergondet, and A. Kheddar, "Collaborative human-humanoid carrying using vision and haptic sensing," in 2014 IEEE international conference on robotics and automation (ICRA). IEEE, 2014, pp. 607-612.

[4] W. Kim, J. Lee, L. Peternel, N. Tsagarakis, and A. Ajoudani, "Anticipatory robot assistance for the prevention of human static joint overloading in human-robot collaboration," IEEE Robotics and Automation Letters, vol. 3, no. 1, pp. 68-75, 2018.

[5] L. Peternel, N. Tsagarakis, and A. Ajoudani, "Towards multi-modal intention interfaces for human-robot co-manipulation," in Intelligent Robots and Systems (IROS), 2016 IEEE/RSJ International Conference on. IEEE, 2016, pp. 2663-2669.
[6] R. Alami, A. Albu-Schäffer, A. Bicchi, R. Bischoff, R. Chatila, A. De Luca, A. De Santis, G. Giralt, J. Guiochet, G. Hirzinger et al., "Safe and dependable physical human-robot interaction in anthropic domains: State of the art and challenges," in EEE/RSJ Int. Conf. on Intelligent Robots and Systems. IEEE, 2006, pp. 1-16.

[7] A. M. Zanchettin, N. M. Ceriani, P. Rocco, H. Ding, and B. Matthias, "Safety in human-robot collaborative manufacturing environments: Metrics and control," IEEE Transactions on Automation Science and Engineering, vol. 13, no. 2, pp. 882-893, 2016.

[8] D. Kulić and E. Croft, "Pre-collision safety strategies for human-robot interaction," Autonomous Robots, vol. 22, no. 2, pp. 149-164, 2007.

[9] C. Esteves, G. Arechavaleta, and J.-P. Laumond, "Motion planning for human-robot interaction in manipulation tasks," in Mechatronics and Automation, 2005 IEEE International Conference, vol. 4. IEEE, 2005, pp. 1766-1771.

[10] W. Kim, M. Lorenzini, P. Balatti, P. D. Nguyen, U. Pattacini, V. Tikhanoff, L. Peternel, C. Fantacci, L. Natale, G. Metta, and A. Ajoudani, "A reconfigurable and adaptive human-robot collaboration framework for improving worker ergonomics and productivity," IEEE Robotics and Automation Magazine, 2019, in press.

[11] A. G. Marin, M. S. Shourijeh, P. E. Galibarov, M. Damsgaard, L. Fritzsch, and F. Stulp, "Optimizing contextual ergonomics models in human-robot interaction," in 2018 IEEE/RSJ International Conference on Intelligent Robots and Systems (IROS). IEEE, 2018, pp. 1-9.

[12] A. Malaisé, P. Maurice, F. Colas, and S. Ivaldi, "Activity recognition for ergonomics assessment of industrial tasks with automatic feature selection," IEEE Robotics and Automation Letters, 2019.

[13] B. Busch, G. Maeda, Y. Mollard, M. Demangeat, and M. Lopes, "Postural optimization for an ergonomic human-robot interaction," in 2017 IEEE/RSJ International Conference on Intelligent Robots and Systems (IROS). IEEE, 2017, pp. 2778-2785.

[14] S. Bevan, T. Quadrello, R. McGee, M. Mahdon, A. Vavrovsky, and L. Barham, "Fit for work? musculoskeletal disorders in the european workforce. the work foundation," 2009.

[15] W. Kim, J. Lee, N. Tsagarakis, and A. Ajoudani, "A real-time and reduced-complexity approach to the detection and monitoring of static joint overloading in humans," in Rehabilitation Robotics (ICORR), 2017 International Conference on. IEEE, 2017, pp. 828-834.

[16] Y. Wu, P. Balatti, M. Lorenzini, F. Zhao, W. Kim, and A. Ajoudani, "A teleoperation interface for loco-manipulation control of mobile collaborative robotic assistant (moca)," IEEE Robotics and Automation Letters, 2018, under review.

[17] A. Ajoudani, S. B. Godfrey, M. Bianchi, M. G. Catalano, G. Grioli, N. Tsagarakis, and A. Bicchi, "Exploring teleimpedance and tactile feedback for intuitive control of the pisa/IIT soft hand," IEEE Transactions on Haptics, vol. 7, no. 2, pp. 203-215, 2014.

[18] Y. Yamamoto, "Control and coordination of locomotion and manipulation of a wheeled mobile manipulators," Ph.D. dissertation, Citeseer, 1994.

[19] A. Dietrich, K. Bussmann, F. Petit, P. Kotyczka, C. Ott, B. Lohmann, and A. Albu-Schäffer, "Whole-body impedance control of wheeled mobile manipulators," Autonomous Robots, vol. 40, no. 3, pp. 505$517,2016$.

[20] C. Ott, Cartesian Impedance Control of Redundant and Flexible-Joint Robots, 01 2008, vol. 49.

[21] M. Lorenzini, W. Kim, E. De Momi, and A. Ajoudani, "A synergistic approach to the real-time estimation of the feet ground reaction forces and centers of pressure in humans with application to human-robot collaboration," IEEE Robotics and Automation Letters, vol. 3, no. 4, pp. 3654-3661, 2018.

[22] A. González, M. Hayashibe, V. Bonnet, and P. Fraisse, "Whole body center of mass estimation with portable sensors: Using the statically equivalent serial chain and a kinect," Sensors, vol. 14, no. 9, pp. 16955-16971, 2014.

[23] L. Peternel, W. Kim, J. Babič, and A. Ajoudani, "Towards ergonomic control of human-robot co-manipulation and handover," in Humanoid Robotics (Humanoids), 2017 IEEE-RAS 17th International Conference on. IEEE, 2017, pp. 55-60.

[24] J. C. E. Van Der Burg, J. H. Van Dieën, and H. M. Toussaint, "Lifting an unexpectedly heavy object: The effects on low-back loading and balance loss," Clinical Biomechanics, vol. 15, no. 7, pp. 469-477, 2000. 